Research, Society and Development, v. 10, n. 13, e334101321319, 2021

(CC BY 4.0) | ISSN 2525-3409 | DOI: http://dx.doi.org/10.33448/rsd-v10i13.21319

\title{
Análise do uso de antimicrobianos em pacientes idosos iternados em um hospital militar em Belém-PA
}

Analysis of the use of antimicrobials in elderly patients hospitalized in a military hospital in BelémPA

Análisis del uso de antimicrobianos en pacientes ancianos hospitalizados en un hospital militar de

Belém-PA

Recebido: 01/10/2021 | Revisado: 09/10/2021 | Aceito: 13/10/2021 | Publicado: 15/10/2021

Ariana Cristina de Oliveira Azulino

ORCID: https://orcid.org/0000-0001-8176-2899

Hospital de Aeronáutica de Belém, Brasil E-mail: ariazulino@hotmail.com

Edilberto Pontes Garcia Júnior

ORCID: https://orcid.org/0000-0002-8279-7919

Hospital de Aeronáutica de Belém, Brasil E-mail: gibajr2005@yahoo.com.br

Keith Brabo Feitosa Tavares

ORCID: https://orcid.org/0000-0003-1233-4537

Hospital de Aeronáutica de Belém, Brasil E-mail: keithfeitosa@hotmail.com

Adriana Vieira Coelho

ORCID: https://orcid.org/0000-0002-7301-8717

Hospital de Aeronáutica de Belém, Brasil

E-mail: avieirac@yahoo.com.br

Priscila Pessoa Diamantino Reis

ORCID: https://orcid.org/0000-0002-9051-9847

Escola Superior da Amazônia, Brasil

E-mail: prisciladiamantino@gmail.com

Bruna Hogla Rodrigues Afonso

ORCID: https://orcid.org/0000-0003-2130-994X

Universidade Federal do Pará, Brasil

E-mail: bruna.afonso@ics.ufpa.br

Thasmyr das Mercês Gonçalves Corrêa

ORCID: https://orcid.org/0000-0001-9100-0118

Universidade Federal do Pará, Brasil

E-mail: cthasmyr@yahoo.com.br

Gabriel da Silva Duarte

ORCID: https://orcid.org/0000-0002-1791-6862

Universidade Federal do Pará, Brasil E-mail: gabriel.duarte@ ics.ufpa.br

Francisco Daniel Queiroz Brito ORCID: https://orcid.org/0000-0003-1095-6615 Universidade Federal do Pará, Brasil E-mail: francisco.brito@ics.ufpa.br

Alessandra Ribeiro Moraes da Silva ORCID: https://orcid.org/0000-0002-8212-0692 Universidade Federal do Pará, Brasil

E-mail: alessandra.moraes.silva@ ics.ufpa.br

José Eduardo Gomes Arruda

ORCID: https://orcid.org/0000-0002-8331-5563

Universidade Federal do Pará, Brasil E-mail: josearruda@ufpa.br

\section{Resumo}

De acordo com a Organização Mundial da Saúde, o envelhecimento da população é um dos grandes desafios a ser enfrentado pela sociedade. A descoberta dos antimicrobianos transformou positivamente a prática clínica, no entanto, a OMS destaca o crescente surgimento de patógenos resistentes estes. O presente estudo buscou analisar o uso de 
ATM em pacientes idosos internados em um hospital militar de Belém-PA, bem como a identificação de possíveis problemas relacionados a estes. Foram incluídos 12 pacientes no estudo, que tiveram uma média de internação de 36,6 dias e 11 antimicrobianos diferentes prescritos. Destes, 89,7\% prescrições apresentaram indicação adequada, dose em $84,5 \%$ e duração do tratamento em 55,2\%. Após a análise de rastreadores, identificou-se que 3 pacientes apresentaram reações adversas a medicamentos (RAM). A presença de pacientes com prescrição de ATM de forma inadequada, quanto a indicação, dose e duração de tratamento, podem estar relacionadas a ausência de protocolos terapêuticos adequados para uso e administração destes. Desta forma, o monitoramento intensivo do uso de ATM na população idosa em ambiente hospitalar é de extrema importância, buscando evitar o surgimento de RAM, responsáveis muitas vezes pela piora na condição clínica destes pacientes e gastos desnecessários. Por fim, é importante destacar que, novos estudos com um número maior de indivíduos são necessários, para comprovar ou não, a utilização inadequada destes medicamentos nesta população.

Palavras-chave: Antimicrobianos; Idosos; Saúde pública.

\begin{abstract}
According to the World Health Organization, population aging is one of the greatest challenges facing society. The discovery of antimicrobials (ATM) positively transformed clinical practice, however, the WHO highlights the growing emergence of resistant pathogens to these. The present study sought to analyze the use of ATMs in elderly patients hospitalized in a military hospital in Belém-PA, as well as the identification of possible problems related to these. Twelve patients were included in the study, who had a mean hospital stay of 36.6 days and 11 different antimicrobials prescribed. Of these, $89.7 \%$ prescriptions had adequate indication, dose in $84.5 \%$ and duration of treatment in 55.2\%. After analyzing the tracers, it was identified that 3 patients had adverse drug reactions (ADR). The presence of patients with inadequate prescription of TMJ, regarding the indication, dose and duration of treatment, may be related to the absence of adequate therapeutic protocols for their use and administration. Thus, intensive monitoring of TMJ use in the elderly population in a hospital environment is extremely important, seeking to avoid the emergence of ADRs, which are often responsible for the worsening of the clinical condition of these patients and unnecessary expenses. Finally, it is important to highlight those further studies with a larger number of individuals are needed, to prove or not, the inappropriate use of these drugs in this population.
\end{abstract}

Keywords: Antimicrobials; Elderly; Public health.

\title{
Resumen
}

Según la Organización Mundial de la Salud, el envejecimiento de la población es uno de los mayores desafíos a los que se enfrenta la sociedad. El descubrimiento de los antimicrobianos (ATM) ha transformado positivamente la práctica clínica, sin embargo, la OMS destaca la creciente aparición de patógenos resistentes a estos. El presente estudio buscó analizar el uso de cajeros automáticos en pacientes ancianos hospitalizados en un hospital militar de Belém-PA, así como la identificación de posibles problemas relacionados con estos. Se incluyeron en el estudio 12 pacientes, que tuvieron una estancia hospitalaria media de 36,6 días y se prescribieron 11 antimicrobianos diferentes. De estos, el 89,7\% prescripciones tenían indicación adecuada, dosis en 84,5\% y duración del tratamiento en 55,2\%. Después de analizar los trazadores, se identificó que 3 pacientes presentaron reacciones adversas a medicamentos (RAM). La presencia de pacientes con prescripción inadecuada de ATM, en cuanto a la indicación, dosis y duración del tratamiento, puede estar relacionada con la ausencia de protocolos terapéuticos adecuados para su uso y administración. Así, la monitorización intensiva del uso de ATM en la población anciana en un entorno hospitalario es de suma importancia, buscando evitar la aparición de RAM, que muchas veces son responsables del empeoramiento de la condición clínica de estos pacientes y gastos innecesarios. Finalmente, es importante resaltar que se necesitan más estudios con un mayor número de individuos, para comprobar o no, el uso inadecuado de estos fármacos en esta población.

Palabras clave: Antimicrobianos; Ancianos; Salud pública.

\section{Introdução}

De acordo com a Organização Mundial da Saúde, o envelhecimento da população é um dos grandes desafios a ser enfrentado pela sociedade (OMS, 2015). A mudança demográfica provoca impacto em todas as políticas públicas, além da saúde, da educação, trabalho, previdência e assistência social. Para enfrentar este processo generalizado e duradouro, é preciso repensar o modelo de atenção à pessoa idosa, incorporando novas estratégias e perspectivas de cuidados (Teixeira et al., 2017).

A descoberta dos antimicrobianos (ATM) transformou positivamente a prática clínica, no entanto, a Organização Mundial de Saúde (OMS) destaca o crescente surgimento de patógenos resistentes a estes. Segundo a OMS a resistência desenvolvida pelos microrganismos corresponde a um grave problema de saúde pública e, portanto, intervenções políticas e 
esforços mundiais devem existir para o controle e implementação de estratégias efetivas para a preservação da vida (WHO, 2019).

No Brasil, a Agência Nacional de Vigilância Sanitária (ANVISA), alinhada com a OMS, cria a diretriz nacional para elaboração de programa de gerenciamento do uso de ATM em serviços de saúde, fornecendo, os elementos fundamentais que devem ser adaptados de acordo com a realidade da população, bem como suas necessidades locais, perfis epidemiológicos e microbiológicos (Brasil, 2016).

As reações adversas a medicamentos (RAM) relacionadas ao seu uso de ATM, são bastante relatadas na literatura. Gallelli et al. (2002) através de um estudo retrospectivo, observaram que cerca de 45\% das RAM identificadas resultavam da utilização de ATM, por serem uma das classes farmacoterapêuticas mais administradas no ambiente hospitalar. A detecção de RAM constitui uma importante medida de morbidade associada a medicamentos e de elevação de custos para o sistema de saúde, visto que tal situação pode levar a um aumento do tempo de internação (Krähenbhül-Melcher et al., 2007). Passarelli (2007) identificou em seu estudo que, no Brasil, os principais fatores de risco para RAM são: o número de diagnósticos, de medicamentos e o uso de medicamentos inapropriados para idosos com base nos critérios de BEERS, estabelecidos pela Sociedade Americana de Geriatria (AGS Beers Criteria ${ }^{\circledR}$ ).

No Brasil, a população idosa entre 2012 e 2017 cresceu 18\%, ultrapassando os 30 milhões de brasileiros (IBGE, 2017). A expectativa de vida dos brasileiros é estimada em 76 anos, existindo uma diferença entre as mulheres e os homens, sendo 79,6 e 72,5 anos, respectivamente. Nesse cenário, a expectativa é que o número de pessoas com 60 anos ou mais praticamente triplique, chegando a 58,2 milhões em 2060 , o equivalente a $25,5 \%$ da população. Tornando-se um desafio as adequações da previdência social e sistema de saúde (IBGE, 2018).

O presente estudo buscou analisar o uso de ATM em pacientes idosos internados em um hospital militar de Belém-PA, bem como a identificação de possíveis problemas relacionados a estes.

\section{Metodologia}

O estudo ocorreu no Hospital de Aeronáutica de Belém (HABE) entre outubro de 2019 e outubro de 2020 e adotou uma abordagem descritiva e prospectiva. Os critérios de inclusão foram pacientes maiores de 60 anos; de ambos os sexos; internados na clínica médica do HABE; que fizeram uso de ATM e que aceitaram participar do estudo, autorizando com o Termo de Consentimento Livre Esclarecido (TCLE) e os de exclusão, pacientes menores de 60 anos; que fizeram uso de ATM para fins de tratamento profilático em cirurgia e os pacientes idosos que fizeram tratamento com ATM não padronizados no hospital. Com relação aos aspectos éticos, este foi submetido à Plataforma Brasil, CAAE: 22140619.6.0000.0018 e aprovado via Parecer Consubstanciado n 3.618.570.

Foi realizada a avaliação das fichas de solicitação de ATM, padronizadas pela instituição e prescrição medicamentosa inicial e nova análise, após resultado de cultura bacteriana e teste de sensibilidade a antimicrobianos (TSA), que eram encaminhados aos laboratórios conveniados ao hospital.

A base de dados Medscape ${ }^{\circledR}$ foi utilizada como ferramenta de referência clínica para análise do uso correto dos ATM, sendo considerada adequada quando a justificativa para a prescrição estava em conformidade com ela. A cada dois dias os dados dos prontuários eram reavaliados, informações como queixas do paciente, justificativas para mudança de esquema ATM e relatos da equipe da assistência eram coletados pelo profissional farmacêutico.

Quanto a identificação de RAM, foram utilizados 17 rastreadores, divididos entre medicamentos, exames laboratoriais e eventos específicos, ajustando-se à realidade local do hospital (Cortés, 2016). Tentou-se estabelecer a relação de causalidade 
entre a administração do fármaco e o surgimento dos eventos utilizando-se o algoritmo de Naranjo (1981). Os critérios de gravidade, seguiram os estabelecidos por Naranjo e Busto (1991).

Para a análise dos dados, procedeu-se a distribuição de frequência absoluta e relativa das variáveis estudadas, organizadas em tabelas e gráficos usando o programa Microsoft Office 365® e a análise estatística foi executada a partir do programa BioEstat@ 5.0, adotando nível de significância de 5\% (Ayres et al., 2007).

\section{Resultados}

No período do estudo, houve 157 internações no HABE, todavia, apenas 12 pacientes aceitaram participar do estudo, o sexo mais frequente, foi o feminino $(66,6 \%)$, e a faixa etária com maior distribuição, foi a de $70-79$ anos $(58,3 \%)$, conforme demonstrados na Tabela 1.

Tabela 1 - Perfil dos pacientes analisados de acordo com sexo e idade.

\begin{tabular}{ccc}
\hline Variável & $\boldsymbol{n}$ & $\boldsymbol{\%}$ \\
\hline Sexo & & $66,7 \%$ \\
Feminino & 8 & $33,3 \%$ \\
Masculino & 4 & $16,7 \%$ \\
Faixa etária & 2 & $58,3 \%$ \\
$60-69$ & 7 & $25 \%$ \\
$70-79$ & 3 & \\
$80+$ & 12 & \\
\hline
\end{tabular}

Fonte: Autores (2021).

Foram utilizados, 11 ATM diferentes, onde a ceftriaxona (Cefalosporina de $3^{\text {a }}$ geração) e o meropeném (Carbapenêmico) foram prescritos para 7 (58,3\%). Quanto à média de dias de utilização, a claritromicina foi utilizada por 21 dias, apesar de ter sido prescrita para apenas 01 paciente, o meropeném e a piperacilina tiveram média de utilização de 20,4 e 20,3 dias, respectivamente. A relação de ATM utilizados por pacientes e média de tempo de utilização estão descritos na Tabela 2. 
Research, Society and Development, v. 10, n. 13, e334101321319, 2021

(CC BY 4.0) | ISSN 2525-3409 | DOI: http://dx.doi.org/10.33448/rsd-v10i13.21319

Tabela 2 - Distribuição de utilização de antimicrobianos por paciente.

\begin{tabular}{ccc}
\hline Antimicrobiano & Pacientes $(\boldsymbol{n}=\mathbf{1 2})$ & Média de uso (dias) \\
\hline Ceftriaxona & $58,3 \%$ & 11,14 \\
Ciprofloxacino & $41,6 \%$ & 8,4 \\
Claritromicina & $8,3 \%$ & 10,5 \\
Clindamicina & $16,6 \%$ & 17 \\
Fluconazol & $16,6 \%$ & 8,5 \\
Metronidazol & $50 \%$ & 20,4 \\
Meropeném & $58,3 \%$ & 7 \\
Oxacilina & $8,3 \%$ & 20,3 \\
Piperacilina/tazobactam & $25 \%$ & 9 \\
Sulfa/trimetropina & $16,6 \%$ & 13,2 \\
Vancomicina & $50 \%$ & 9 \\
\hline
\end{tabular}

Fonte: Autores (2021).

A média de internação foi de 36,6 dias, e utilização de ATM por paciente, foi de 3,4 e em 58,3\% dos pacientes, foi coletado amostra para realização de cultura e TSA. A relação detalhada está descrita na Tabela 3.

Tabela 3 - Distribuição dos pacientes por tempo de internação, ATM prescritos e realização de teste de cultura/TSA.

\begin{tabular}{|c|c|c|}
\hline Variável & $n$ & $\%$ \\
\hline \multicolumn{3}{|l|}{ Tempo de internação } \\
\hline $1-9$ & 3 & $25 \%$ \\
\hline $10-19$ & 2 & $16,7 \%$ \\
\hline $20-29$ & 1 & $8,3 \%$ \\
\hline $30-39$ & 2 & $16,7 \%$ \\
\hline $40-49$ & 1 & $8,3 \%$ \\
\hline $50-59$ & 1 & $8,3 \%$ \\
\hline $60+$ & 2 & $16,7 \%$ \\
\hline \multicolumn{3}{|l|}{ ATM prescritos } \\
\hline $1-3$ & 7 & $58,3 \%$ \\
\hline $4-6$ & 4 & $33,3 \%$ \\
\hline $7-10$ & 1 & $8,3 \%$ \\
\hline \multicolumn{3}{|l|}{ Realização de cultura/TSA } \\
\hline Sim & 7 & $58,3 \%$ \\
\hline Não & 5 & $41,7 \%$ \\
\hline
\end{tabular}

Fonte: Autores (2021). 
Quanto a indicação terapêutica, 89,7\% prescrições de ATM estavam adequadas, que apresentaram dose terapêutica e duração do tratamento adequadas em $84,5 \%$ e 55,2\% respectivamente. A adequação da indicação de uso, dose e tempo de tratamento estão descritos na Tabela 4.

Tabela 4 - Adequação de indicação, dose e tempo de utilização $(n=58)$.

\begin{tabular}{|c|c|c|c|c|c|c|c|c|c|c|}
\hline \multirow[t]{2}{*}{ ATM } & \multicolumn{2}{|c|}{$\begin{array}{c}\text { Indicação } \\
\text { Terapêutica } \\
\end{array}$} & \multicolumn{3}{|c|}{ Dose } & \multicolumn{3}{|c|}{$\begin{array}{l}\text { Duração do } \\
\text { Tratamento }\end{array}$} & \multicolumn{2}{|c|}{$\begin{array}{c}\text { Tratamento } \\
\text { Empírico }\end{array}$} \\
\hline & $\mathbf{P A}$ & $\mathbf{S} / \mathbf{I}$ & PA & + & - & PA & + & - & Sim & Não \\
\hline Ceftriaxona & 7 & 1 & 7 & & & 4 & & 3 & 7 & 1 \\
\hline Ciprofloxacino & 4 & 1 & 3 & & 1 & 1 & & 3 & 3 & 2 \\
\hline Claritromicina & 2 & & 2 & & & 1 & 1 & & 1 & 1 \\
\hline Clindamicina & 2 & & 2 & & & 1 & 1 & & 2 & \\
\hline Fluconazol & 4 & & 4 & & & 3 & & 1 & 2 & 2 \\
\hline Metronidazol & 6 & 1 & 6 & & & 5 & & 1 & 5 & 2 \\
\hline Meropeném & 11 & 1 & 9 & 1 & 1 & 6 & 5 & & 7 & 5 \\
\hline Oxacilina & & 1 & & & & & & & & 1 \\
\hline Piper/tazobactam & 7 & & 7 & & & 5 & 2 & & 6 & 1 \\
\hline Sulfa/trimetropina & 2 & & 2 & & & 2 & & & 1 & 1 \\
\hline Vancomicina & 7 & 1 & 7 & & & 4 & 3 & & 4 & 4 \\
\hline
\end{tabular}

Legenda: PA - prescrições adequadas; S/I - prescrições sem recomendação de uso clínico; (+) - dose e duração de tratamento acima do preconizado; (-) - subdose e duração de tratamento menor do que o preconizado. Fonte: Autores (2021).

Foram realizadas 14 coletas de material clínico para cultura em 7 pacientes, com positividade em 7 amostras. Observouse predominância de bactérias gram negativas, como Pseudomonas aeruginosas e Stenotrophomonas maltophilia (28,6\%) e fungos do gênero Candida sp (42,9\%), sendo que em um mesmo paciente foram identificadas duas espécies, a Candida albicans e a Candida glabrata. As amostras coletadas foram de sítios diferentes, e possuíam perfis de sensibilidade a ATM distintas, como demonstrado no Quadro 1. 
Quadro 1 - Relação de patógenos isolados nas culturas dos 7 pacientes e resultado do teste de sensibilidade a antimicrobianos.

\begin{tabular}{|c|c|c|c|c|}
\hline \multirow{2}{*}{ Patógeno } & \multirow{2}{*}{$\begin{array}{c}n=7 \\
(100 \%)\end{array}$} & \multicolumn{2}{|c|}{ Resultado do TSA } & \multirow{2}{*}{ Local de coleta } \\
\hline & & Sensível & Resistente & \\
\hline Candida parapsilosis & $14,3 \%$ & $\begin{array}{l}\text { Fluconazol } \\
\text { Voriconazol, } \\
\text { Caspofungina } \\
\text { Micafungina } \\
\text { Anfotericina B } \\
\text { Fluocitosina }\end{array}$ & & Urocultura \\
\hline $\begin{array}{l}\text { Pseudomonas } \\
\text { aeruginosas }\end{array}$ & $28,6 \%$ & $\begin{array}{l}\text { Amicacina, } \\
\text { Ciprofloxacino } \\
\text { Colistina } \\
\text { Gentamicina } \\
\text { Imipeném } \\
\text { Meropeném } \\
\text { Vancomicina } \\
\end{array}$ & $\begin{array}{c}\text { Cefepime } \\
\text { Ceftazidima } \\
\text { Piperacilina/tazobactam } \\
\text { Tigeciclina }\end{array}$ & $\begin{array}{c}\text { Hemocultura } \\
\text { Secreção de } 3^{\circ} \\
\text { poodáctilo direito }\end{array}$ \\
\hline Candida glabrata & $14,3 \%$ & $\begin{array}{l}\text { Caspofungina } \\
\text { Micafungina } \\
\text { Anfotericina B } \\
\text { Fluocitosina } \\
\end{array}$ & & Hemocultura \\
\hline Candida albicans & $14,3 \%$ & $\begin{array}{c}\text { Fluconazol } \\
\text { Voriconazol } \\
\text { Caspofungina } \\
\text { Micafungina } \\
\text { Anfotericina B } \\
\text { Fluocitosina } \\
\end{array}$ & & Urocultura \\
\hline $\begin{array}{l}\text { Stenotrophomonas } \\
\text { maltophilia }\end{array}$ & $28,6 \%$ & $\begin{array}{c}\text { Levofloxacino } \\
\text { Sulfametoxazol/trimetropina }\end{array}$ & $\begin{array}{l}\text { Ceftazidima } \\
\text { Imipeném } \\
\text { Meropeném }\end{array}$ & Secreção traqueal \\
\hline Morganella morganii & $14,3 \%$ & $\begin{array}{c}\text { Amicacina } \\
\text { Ampicilina/sulbactam } \\
\text { Cefepima } \\
\text { Ceftazidima } \\
\text { Ceftriaxona } \\
\text { Ciprofloxacino } \\
\text { Ertapenem } \\
\text { Gentamicina } \\
\text { Meropeném } \\
\text { Piperacilina/tazobactam }\end{array}$ & Ampicilina & $\begin{array}{l}\text { Secreção de } \\
\text { ferida pé } \\
\text { esquerdo }\end{array}$ \\
\hline
\end{tabular}

Fonte: Autores (2021).

De acordo com o perfil de sensibilidade aos ATM foram estabelecidos 7 esquemas terapêuticos, sendo 85,7\% considerados adequados. Com relação à duração do tratamento, 42,8\% estavam adequadas e 57,2\% estavam menores do que os preconizados ou sem indicação de uso. O detalhamento dos esquemas terapêuticos utilizados após resultado de cultura bacteriana e TSA, está descrito no Quadro 2. 
Research, Society and Development, v. 10, n. 13, e334101321319, 2021

(CC BY 4.0) | ISSN 2525-3409 | DOI: http://dx.doi.org/10.33448/rsd-v10i13.21319

Quadro 2 - Esquema terapêutico após resultado do teste de sensibilidade a antimicrobianos.

\begin{tabular}{|c|c|c|c|c|c|c|c|}
\hline \multirow{2}{*}{ ATM } & \multicolumn{3}{|c|}{ Indicação terapêutica } & \multicolumn{4}{|c|}{ Posologia } \\
\hline & Infecção & \begin{tabular}{|l|} 
Patógeno \\
\end{tabular} & Adequado & Dose & Adequado & Duração & Adequado \\
\hline Meropenem & \multirow{2}{*}{$\begin{array}{l}\text { ICS (foco } \\
\text { pulmonar) }\end{array}$} & \multirow{2}{*}{$\begin{array}{l}\text { Psedomonas } \\
\text { aeruginosas }\end{array}$} & Sim & $2 \mathrm{~g} 8 / 8 \mathrm{~h}$ & + & 14 dias & Sim \\
\hline Vancomicina & & & Sim & $\operatorname{lg~} 12 / 12 \mathrm{~h}$ & Sim & 14 dias & Sim \\
\hline Fluconazol & \multirow{3}{*}{$\begin{array}{l}\text { ICS (foco } \\
\text { pulmonar) }\end{array}$} & \multirow{3}{*}{$\begin{array}{l}\text { C. glabrata } \\
\text { C. albicans }\end{array}$} & Sim & $200 \mathrm{mg} 12 / 12 \mathrm{~h}$ & Sim & 14 dias & Sim \\
\hline $\begin{array}{l}\text { Piperacilina/ } \\
\text { Tazobactam }\end{array}$ & & & Sim & $4,5 \mathrm{mg} 6 / 6 \mathrm{~h}$ & Sim & 4 dias & - \\
\hline Meropenem & & & Sim & $1 \mathrm{~g} 8 / 8 \mathrm{~h}$ & Sim & 7 dias & - \\
\hline Meropenem & \multirow[b]{3}{*}{ PNM } & \multirow{3}{*}{$\begin{array}{l}\text { Stenotrophomonas } \\
\text { maltophilia }\end{array}$} & Sim & $1 \mathrm{~g} 8 / 8 \mathrm{~h}$ & Sim & 10 dias & Sim \\
\hline Vancomicina & & & Sim & $\operatorname{lg~} 12 / 12 \mathrm{~h}$ & Sim & 7 dias & - \\
\hline $\begin{array}{c}\text { Sulfametoxa } \\
\text { zol/ } \\
\text { Trimetropina }\end{array}$ & & & Sim & 400/80mg 8/8h & Sim & 7 dias & Sim \\
\hline $\begin{array}{c}\text { Ciprofloxaci } \\
\text { no }\end{array}$ & Pé diabético & P. aeruginosas & Sim & $500 \mathrm{mg} 12 / 12 \mathrm{~h}$ & Sim & 7 dias & - \\
\hline $\begin{array}{c}\text { Metronidazo } \\
1\end{array}$ & \multirow[t]{2}{*}{ Pé diabético } & \multirow[t]{2}{*}{ Morganella morganii } & Sim & $500 \mathrm{mg} 8 / 8 \mathrm{~h}$ & Sim & 7 dias & - \\
\hline Oxacilina & & & $\mathrm{S} / \mathrm{I}$ & $2 \mathrm{~g} \mathrm{4/4h}$ & $\mathrm{S} / \mathrm{I}$ & 7 dias & $\mathrm{S} / \mathrm{I}$ \\
\hline Vancomicina & IRAS & Sem identificação & Sim & $\operatorname{lg~} 12 / 12 \mathrm{~h}$ & Sim & 10 dias & Sim \\
\hline $\begin{array}{l}\text { Sulfametoxa } \\
\text { zol/ } \\
\text { Trimetropina }\end{array}$ & Leucocitose & $\begin{array}{c}\text { Stenotrophomonas } \\
\text { maltophilia }\end{array}$ & Sim & $800 / 160 m g ~ 8 / 8 h$ & Sim & 7 dias & Sim \\
\hline
\end{tabular}

Legenda: (+) dose acima do preconizado; (-) tempo menor do que o preconizado; S/I: sem indicação terapêutica. Fonte: Autores (2021).

Com relação as RAM, foram identificadas 18 rastreadores (Quadro 3), que após análise, demonstraram 3 pacientes com RAM, onde o primeiro foi definido como reação provável e de gravidade leve, o segundo como provável e moderada e o terceiro como possível e de gravidade moderada. O detalhamento dos casos de RAM de acordo com os rastreadores de busca e segundo suas respectivas classificações estão descritos na Tabela 5. 
Research, Society and Development, v. 10, n. 13, e334101321319, 2021

(CC BY 4.0) | ISSN 2525-3409 | DOI: http://dx.doi.org/10.33448/rsd-v10i13.21319

Quadro 3 - Rastreadores utilizados para busca de reações adversas a medicamentos.

\begin{tabular}{|l|c|c|}
\hline Tipo de rastreador & Rastreador & Quantidade de pacientes \\
\hline \multirow{4}{*}{ Medicamento } & Dexametasona & 3 \\
\cline { 2 - 3 } & Hidrocortisona & 1 \\
\cline { 2 - 3 } & Lactulose & 1 \\
\cline { 2 - 3 } & Loratadina & 7 \\
\cline { 2 - 3 } & Omeprazol & 8 \\
\cline { 2 - 3 } & Ondansetrona & 1 \\
\cline { 2 - 3 } & Prometazina & 5 \\
\cline { 2 - 3 } & Ranitidina & 6 \\
\hline \multirow{5}{*}{ Evento específico } & Saccharomyces boulardii & 2 \\
\cline { 2 - 3 } & Diarreia & 1 \\
\cline { 2 - 3 } & Prurido & 1 \\
\cline { 2 - 3 } & Rash cutâneo & 2 \\
\cline { 2 - 3 } & Mudança de dose & 1 \\
\cline { 2 - 3 } & Náusea/vômito & 1 \\
\cline { 2 - 3 } & Menção de hipotensão & \\
\hline \multirow{5}{*}{ Exames laboratoriais } & Alteração de creatinina & 1 \\
\hline
\end{tabular}

Fonte: Autores (2021).

Tabela 5 - Detalhamento dos casos suspeitos de reações adversas a medicamentos.

\begin{tabular}{|c|c|c|c|c|c|}
\hline \multirow{2}{*}{$\begin{array}{l}\text { Tempo de } \\
\text { internação } \\
\text { (dias) }\end{array}$} & \multirow{2}{*}{$\begin{array}{c}\text { No de ATM }^{\circ} \text { utilizados }\end{array}$} & \multirow{2}{*}{ Rastreador } & \multirow{2}{*}{$\begin{array}{l}\text { Medicamento } \\
\text { suspeito }\end{array}$} & \multicolumn{2}{|c|}{ Classificação de Naranjo } \\
\hline & & & & Causalidade & Gravidade \\
\hline 112 & 6 & $\begin{array}{c}\text { Rash } \\
\text { Prurido } \\
\text { Prometazina }\end{array}$ & Vancomicina & Provável & Leve \\
\hline 81 & 7 & $\begin{array}{c}\text { Diarreia } \\
\text { Saccharomyces }\end{array}$ & Fluconazol & Provável & Moderada \\
\hline 49 & 6 & $\begin{array}{c}\text { Diarreia } \\
\text { Saccharomyces }\end{array}$ & $\begin{array}{l}\text { Sulfametoxazol/ } \\
\text { trimetropina }\end{array}$ & Possível & Moderada \\
\hline
\end{tabular}

Fonte: Autores (2021).

\section{Discussão}

As consequências do alto consumo de ATM, diferentemente de outros tipos de medicamentos, está associado ao aumento de microrganismos resistentes, não afetando somente o paciente que está com a infecção, mas a comunidade hospitalar. Podendo ocorrer um aumento de gastos com saúde, que vão desde uma maior permanência do paciente no hospital, englobando gastos com exames mais sofisticados e procedimentos, utilização de ATM mais potentes até gastos indiretos representados pela perda de produtividade do paciente (De With et al., 2016; Larson et al., 2017).

Quando analisamos a internação desta população, Souza (2015) demonstrou a predominância de pacientes do sexo feminino em um Hospital Universitário de Belém-PA, conforme relatado por Rabelo et al. (2010), encontraram proporção semelhante em um Hospital Universitário de Minas Gerais, que corroboram com os encontrados neste estudo, onde 66,7\% dos pacientes eram do sexo feminino. 
Research, Society and Development, v. 10, n. 13, e334101321319, 2021

(CC BY 4.0) | ISSN 2525-3409 | DOI: http://dx.doi.org/10.33448/rsd-v10i13.21319

Com relação ao número de ATM utilizado por paciente, as proporções encontradas estão de acordo com o estudo realizado no interior do Rio Grande do Sul, onde dos 1.166 pacientes que usaram ATM, 92,6\% utilizaram até dois. (Spiandorello et al., 2006). Em um estudo realizado em Belém-PA, o consumo de mais de um tipo de ATM foi frequente, com a média de 3,16 e a mediana de 3 por paciente. Alguns pacientes utilizaram 9 classes diferentes de ATM durante sua internação. Situação semelhante a encontrada no presente estudo onde 8,3\% dos pacientes utilizaram de 7-10 ATM (Menezes, 2013).

O meropeném e a vancomicina foram os que tiveram maior uso inadequado na duração do tratamento $(41,7 \%)$ e indicação de uso (37,5\%). Seis ATM foram utilizados sem indicação clínica prevista em protocolos clínicos, de acordo com o Medscape ${ }$, são eles: ceftriaxona, ciprofloxacino, meropeném, metronidazol, oxacilina e vancomicina, o que dificultou a avaliação da adequação da dose e tempo tratamento. Em relação ao uso da oxacilina em um dos pacientes, chama a atenção o fato de ter sido realizado a Cultura e TSA, sendo identificado o patógeno Morganella morganii, que de acordo com o TSA, não apresentava sensibilidade ao antimicrobiano prescrito. Mesmo após a intervenção junto ao médico prescritor, este manteve a prescrição do referido ATM.

Segundo Kollof (2000) e Chastre (2002), a utilização de ATM sem a identificação adequada de microrganismos, pode ser considerada como uso de forma empírica. Em estudo desenvolvido por Mendonça et al. (2009), em três hospitais da rede sentinela de médio e grande porte, constataram que a indicação do uso empírico de ATM ocorreu em $93 \%$ das prescrições. No presente estudo, apesar de 58,3\% dos pacientes terem feito coleta de material clínico para realização de cultura e TSA, 65,5\% dos esquemas terapêuticos foram definidos de forma empírica.

Em relação ao tempo de permanência, a média neste estudo, foi de 36,6 dias, superior a encontradas em estudos semelhantes, que variaram de 10 a de 21,9 (Maués et al., 2007; Carneiro et al., 2011). A diferença entre as médias pode estar relacionada ao tipo de paciente atendido em cada hospital, às especialidades médicas disponíveis e aos tratamentos executados, podendo oscilar em diferentes períodos do ano de acordo com as causas da internação (Spiandorello et al., 2006). Um estudo com 186 pacientes em um hospital em Santo André- SP, constatou que quanto maior o tempo de permanência hospitalar, maior o número de reações adversas (Passarelli et al., 2007).

As RAM cutâneas são comuns à diversos medicamentos, incluindo os antimicrobianos. A identificação do fármaco responsável torna-se difícil devido ao elevado número de medicamentos administrados em pacientes geriátricos e ao fato destas reações só se desenvolverem dias ou semanas após o início da terapêutica (Lin, et al. 2014). Os agentes mais frequentemente associados são os ß-lactâmicos, sulfonamidas, fluoroquinolonas e vancomicina. No presente estudo foi identificado a ocorrência de três RAM, uma possivelmente associada à vancomicina. Os eventos mais relacionados ao uso da vancomicina são as reações de hipersensibilidade, como erupções de pele, febres e dermatite esfoliativa, podendo ocorrer em 5\% dos pacientes (Micromedex, 2015). Em um estudo realizado no Hospital das Clínicas da Faculdade de Medicina de Ribeirão Preto-SP, o período de tratamento com a vancomicina variou de 1 a 70 dias com taxa de incidência de RAM de 7,18\%, semelhante com o encontrado neste estudo, de 1 a 79 dias, com incidência de 8,3\% (Queiroz Netto, 2010).

Os rastreadores são instrumentos importantes para identificar RAM, sendo possível mensurá-las (Rozenfeld, 2013). No presente estudo, os rastreadores mais frequentes foram a ondansetrona $(44,4 \%)$, omeprazol $(38,9 \%)$ e ranitidina $(2,8 \%)$, resultado semelhante ao encontrado por Giordani et al. (2012), onde os rastreadores mais presentes foram os antieméticos, que compreenderam a $20,6 \%$.

Um possível viés do estudo foi o número reduzido de pacientes. Outra dificuldade foi a ausência em prontuário, da justificativa para solicitação de mudança do esquema terapêutico de alguns pacientes. Tais informações são de relevância para 
um monitoramento efetivo e para as tomadas de decisões necessárias no manejo do tratamento do paciente, especialmente o idoso.

\section{Considerações Finais}

A presença de pacientes com prescrição de ATM de forma inadequada, quanto a indicação, dose e duração de tratamento, podem estar relacionadas a ausência de protocolos terapêuticos adequados para uso e administração destes. Desta forma, o monitoramento intensivo do uso de ATM na população idosa em ambiente hospitalar é de extrema importância, buscando evitar o surgimento de RAM, responsáveis muitas vezes pela piora na condição clínica destes pacientes e gastos desnecessários. Por fim, é importante destacar que, novos estudos com um número maior de indivíduos são necessários, para comprovar ou não, a utilização inadequada destes medicamentos nesta população.

\section{Referências}

Ayres, M., Ayres Jr, M., Ayres, L. D., \& Santos, S. A. A. (2007). BioEstat 5.0.: aplicações estatísticas nas áreas das Ciências Biomédicas. Sociedade Civil Mamirauá: Belém, Pará-Brasil. 324p. Aplicações estatísticas nas áreas das ciências biomédicas. Ong Mamirauá. Belém, PA.

Brasil. (2016). Agência Nacional de Vigilância Sanitária. Programa Nacional de Prevenção e Controle de Infecções Relacionadas à Assistência à Saúde.

Chastre, J., \& Fagon, J. Y. (2002). Ventilator-Associated Pneumonia. State of The Art. Am J Respir Crit Care Med. v. 165, n 7, p. 867-903. https://doi.org/10.1164/ajrccm.165.7.2105078.

Cortés, S. D. C. (2016). Busca de reações adversas a medicamentos em pacientes internados em Clínica Médica usando rastreadores. Dissertação (Mestrado) Faculdade de Saúde Pública da Universidade de São Paulo, São Paulo.

Carneiro, M., Ferraz, T., Bueno, M., Koch, E. B., Foresti, C., Lena, F. V., Machado, A. J., Rauber, M. J., Krummenaauer, C. E., \& Lazaroto, M. D. (2011). O uso de antimicrobianos em um hospital de ensino: uma breve avaliação. Revista da Associação Médica Brasileira. Volume 57, Issue 4, Pages 421-424. https://doi.org/10.1590/S0104-42302011000400016.

De With, K., Allerberger, F., Amann, S. et al. (2016). Strategies to enhance rational use of antibiotics in hospital: a guideline by the German Society for Infectious Diseases. Infection 44, 395-439 (2016). https://doi.org/10.1007/s15010-016-0885-z

Gallelli, L., Ferreri, G., Colosimo, M., Pirritano, D., Guadagnino, L., Pelaia, G., Maselli, R., \& De Sarro, G. B. (2002). Adverse drug reactions to antibiotics observed in two pulmonology divisions of catanzaro, Italy: a six-year retrospective study. Pharmacol Res.46(5):395-400. doi:https://10.1016/s1043661802002104. PMID: 12419643.

Giordani, F. Rozenfeld, S. Gelena, O. M. F. D., \& Joelma, V. S. G. L. (2012). Suto TerencioLuciane de Fátima CaldeiraLuiz Carlos Gonçalves de Andrade. Vigilância de eventos adversos a medicamentos em hospitais: aplicação e desempenho de rastreadores. Rev Bras Epidemiol. v.15, n. 3, p. 455-67. https://doi.org/10.1590/S1415-790X2012000300002.

Instituto Brasileiro de Geografia e Estatística (IBGE). (2017). Tábua completa de mortalidade para o Brasil - 2017: breve análise da evolução da mortalidade no Brasil. Rio de Janeiro: IBGE, 2018. 28 p.

Kollef, M. (2000). Inadequate antimicrobial treatment: an important determinant of outcome for hospitalized patients. CID. v. 31, n. suppl_4, p. 131-8. https://doi.org/10.1378/chest.115.2.462.

Krahenbuhl-Melcher, A., et al. (2007). Drug-related problems in hospital: a review of the recente literature. Drug Saf. v.30, n. 5, p. 379-407, 2007. https://doi.org/10.2165/00002018-200730050-00003

Larson, E. L., Quiros, D., Giblin, T., \& Lin, S. (2007). Relationship of antimicrobial control policies and hospital and infection control characteristics to antimicrobial resistance rates. American journal of critical care: an official publication, American Association of Critical-Care Nurses, 16(2), 110-120. http://dx.doi.org/10.4037/ajcc2007.16.2.110.

Lin, Y. F., Yang, C. H., Sindy, H., Lin, J. Y., Rosaline, C. Y. H., Tsai, Y. C., Wu, T. S., Huang, C. T., Kao, K. C., Hu, H. C., Chiu, C. H., Hung, S. I., \& Chung, W. H. (2014). Severe cutaneous adverse reactions related to systemic antibiotics. Clin Infect Dis. (10):1377-85. https://doi.org/10.1093/cid/ciu126.

Maues, C. R., et al. (2007). Epidemiologia de idosos internados na enfermaria de clínica médica de hospital público. Rev. Para. Med., Belém, v. 21, n. 3, p. 3136, set. 2007. <http://scielo.iec.gov.br/scielo.php?script=sci_arttext\&pid=S0101-59072007000300005\&lng=pt\&nrm=iso>.

Menezes, C. R. (2013). Monitorização do uso de ATM como estratégia de combate à resistência bacteriana em uma UTI de adultos. Dissertação (Mestrado) Instituto de Ciências Biológicas, Programa de Pós-Graduação em Biologia de Agentes Infecciosos e Parasitários, Universidade Federal do Pará, Belém, 2013. 
Research, Society and Development, v. 10, n. 13, e334101321319, 2021

(CC BY 4.0) | ISSN 2525-3409 | DOI: http://dx.doi.org/10.33448/rsd-v10i13.21319

Naranjo, C. A., \& Busto, U. (1991). Reações Adversas às Drogas. In: Kalant, H.; Roschlau, W.H.E. Princípios de Farmacologia Médica. Rio de Janeiro: Guanabara Koogan. p.537-542.

Naranjo, C. A., et al. (1981). A Method for estimating the probability of adverse drug reactions. Clinical. Pharmacology \& Therapeutics. v. 30, n. 2, p.239245.

Organização Mundial da Saúde. (2005). Envelhecimento ativo: uma política de saúde. Brasília, OPAS; 2005.

Passarelli, M. C., Filho, W. J., \& Figueras, A. (2007). Adverse drug reactions in a elderly hospitalized population: inappropriate prescription is a leading cause. Drugs Aging. v. 22, n. 9, p. 767-77. https://doi.org/10.2165/00002512-200522090-00005.

Passarelli, M. C. G., Filho, W. F. (2007). Reações Adversas a Medicamentos em Idosos: Como Prevê-las? Einstein. São Paulo, v. 5 , n. 3, p. 246 - 251.

Queiroz, M. U. N. (2010). Farmacovigilância: monitorização intensiva de vancomicina em pacientes hospitalizados. Dissertação (Mestrado em Medicamentos e Cosméticos) - Faculdade de Ciências Farmacêuticas de Ribeirão Preto, Universidade de São Paulo; 2010. doi:https://10.11606/D.60.2010.tde-29032012110351

Rabelo, L. P. O., Vieira, M. A., Caldeira, A. P., Costa, S. M. (2010). Perfil de idosos internados em um hospital universitario / Perfil de ancianos hospitalizados en un hospital universitario / Profile of elderly hospitalized in a university hospital. REME. V. 14, n. 3, p. 293-300.

Rozenfeld, S., Giordani, F., Coelho, S. (2013). Eventos adversos a medicamentos em hospital terciário: estudo piloto com rastreadores. Revista de Saúde Pública, 47(6),1102-1111. ISSN: 0034-8910. https://www.redalyc.org/articulo.oa?id=67240209009

Souza, N., Oliveira, G., Girão, A., Souza, L., Maniva, S., \& Freitas, C. (2015). Adoecimento por hipertensão arterial e Diabetes Mellitus: concepções de um grupo de pacientes hospitalizados [Conceptions of illness from hypertension and Diabetes Mellitus among a group of hospital inpatients]. Revista Enfermagem UERJ, 23(1), 52-57. doi:https://doi.org/10.12957/reuerj.2015.15579

Spiandorello, W. P., et al. (2006). Use of multiple antimicrobial drugs by clinical patients: a prognostic index of hospital mortality? Clinics. v. 61, p 15-20. https://doi.org/10.1590/S1807-59322006000100004.

Teixeira, J. J. M., et al. (2017). Perfil de internação de idosos. Rev Soc Bras Clin Med. v. 15, n 1, p. 15-20.

Who, et al. (2019). Antimicrobial stewardship programmes in health-care facilities in low-and middle-income countries: a WHO practical toolkit. 2019. <https://apps.who.int/iris/bitstream/handle/10665/329404/9789241515481-eng.pdf?sequence=1\&isAllowed=y> 